\title{
Competitive evolution of the fine contrast modulation and CuPt ordering in InGaP/GaAs layers
}

\author{
A. Diéguez, F. Peiró, ${ }^{\text {a) }}$ A. Cornet, and J. R. Morante \\ EME, Enginyeria i Materials Electronics, Departament de Física Aplicada i Electrònica, \\ Avenida Diagonal 645-647, 08028 Barcelona, Spain \\ F. Alsina ${ }^{\text {b) }}$ and J. Pascual \\ Dept. Física, Universitat Autònoma de Barcelona, 08193 Bellaterra, Spain
}

(Received 8 April 1996; accepted for publication 18 June 1996)

\begin{abstract}
We use transmission electron microscopy to characterize the morphology of InGaP epitaxial layers grown by metal-organic vapor-phase epitaxy over misoriented GaAs (001) substrates, with a cutoff angle in a range from $0^{\circ}$ to $25^{\circ}$. The occurrence of phase separation and CuPt-type ordered superstructures has been observed. The most ordered configuration has been found to appear in layers grown on $2^{\circ}$ off substrates, and the strength of order decreases with increasing the misorientation angle beyond $\alpha=2^{\circ}$. Conversely, whereas the phase separation is less evident in the layer grown at $2^{\circ}$, the sample grown with a misorientation of $25^{\circ}$ exhibits the most phase separated configuration. The completion between these two phenomena is discussed depending on the misorientation angle. (C) 1996 American Institute of Physics. [S0021-8979(96)08218-7]
\end{abstract}

\section{INTRODUCTION}

The growth of ternary and quaternary multilayer systems of $\mathrm{Ga}_{x} \mathrm{In}_{1-x} \mathrm{As}_{y} \mathrm{P}_{1-y}$ of any combination of $x$ and $y$ is of great technological interest for electronics and optoelectronics. By varying $x$ and $y$ independently an accurate control of the band-gap energy and lattice parameter can be obtained. Structurally, these materials consist of two interpenetrating fcc units which are displaced from each other by $1 / 4\langle 111\rangle$. One of the units is occupied by group-III atoms, the group-V atoms being located on the other. Although the majority of applications requires a high-quality homogeneous material with the atoms distributed in a random way, there are two main features that reveal that the epitaxial layers are inhomogeneous on a microscopic scale.

First, the majority of these alloys is predicted to be thermodynamically unstable in the bulk and epitaxial forms, exhibiting miscibility gaps and then showing a tendency toward clustering and phase separation when grown at growth conditions within this gap. ${ }^{1,2}$ Electron microscopy studies have given experimental evidence of miscibility gaps, revealing the existence of composition modulations and clustering from phase separation in epitaxial layers. ${ }^{3-5}$

Second, energy minimization calculations using firstprinciples local density, ${ }^{6}$ performed on $\operatorname{In}_{x} \mathrm{Ga}_{1-x} \mathrm{P}$, have predicted that certain ordered intermediate phases could be thermodynamically stable at low temperature. These ordered phases were concluded to be stable since they can simultaneously accommodate the different $\mathrm{GaP}$ and $\mathrm{InP}$ bond lengths in the alloy in a coherent fashion, introducing less strain than would arise in a random alloy. Since Murgatroyd et al. ${ }^{7}$ reported the first CuPt-type ordering in III-V alloys, this type of ordering has been observed in a wide range of ternary and quaternary alloys (see Norman $e t a l .{ }^{8}$ for a re-

\footnotetext{
${ }^{a}$ Electronic mail: paqui@iris1.fae.ub.es

${ }^{b}$ Present address: National Renewable Energy Laboratory, 16-17 Cole Boulevard, Golden, CO 80401.
}

view), especially in the InGaP system grown by metalorganic chemical-vapor deposition (MOCVD). The use of the regular solution approximation predicts that spinodal decomposition and long-range atomic ordering are mutually exclusive in epitaxially grown group-III-V semiconductors. ${ }^{9}$ A positive enthalpy of mixing $\Delta H_{m}$ is expected to induce spinodal decomposition of the alloy, whereas a negative $\Delta H_{m}$ would favor the ordering of the alloy. This approximation does not account for some observations of ordering made in alloys that have also shown spinodal decomposition. ${ }^{10-13}$ One of the possible explanations of the nonaccordance is that thermodynamic calculations have only considered equilibrium structures whereas molecular-beam epitaxy (MBE) and MOCVD are nonequilibrium growth processes, and furthermore growth occurs at the crystal surface, suggesting that a surface energy term should be included in the thermodynamic calculations. However, the introduction of thermodynamic stability criterium at the surface is not yet enough to explain the maintenance of ordering when growth continues ${ }^{14-16}$ and the influence of the growth conditions on the atomic surface diffusion has also been considered. ${ }^{17,18} \mathrm{In}$ this situation, most of the experimental work done until now has focused its interest in the study of the influence of growth conditions on the presence of order assuming that the phase separation is concomitantly present in the structure and obviating the interesting information that a simultaneous study of the evolution of both structures with the growth conditions could give.

In this work we use transmission electron microscopy (TEM) and transmission electron diffraction (TED) techniques to study the influence of the substrate misorientation on the existence of ordering and phase separation in $\mathrm{InGaP}$ layers grown on GaAs substrates. Our results show that, although both structures coexist in our samples, they are competitive; the predominance of one or the other being influenced by the surface reconstruction in concordance with the substrate misorientation angle and growth temperature. 

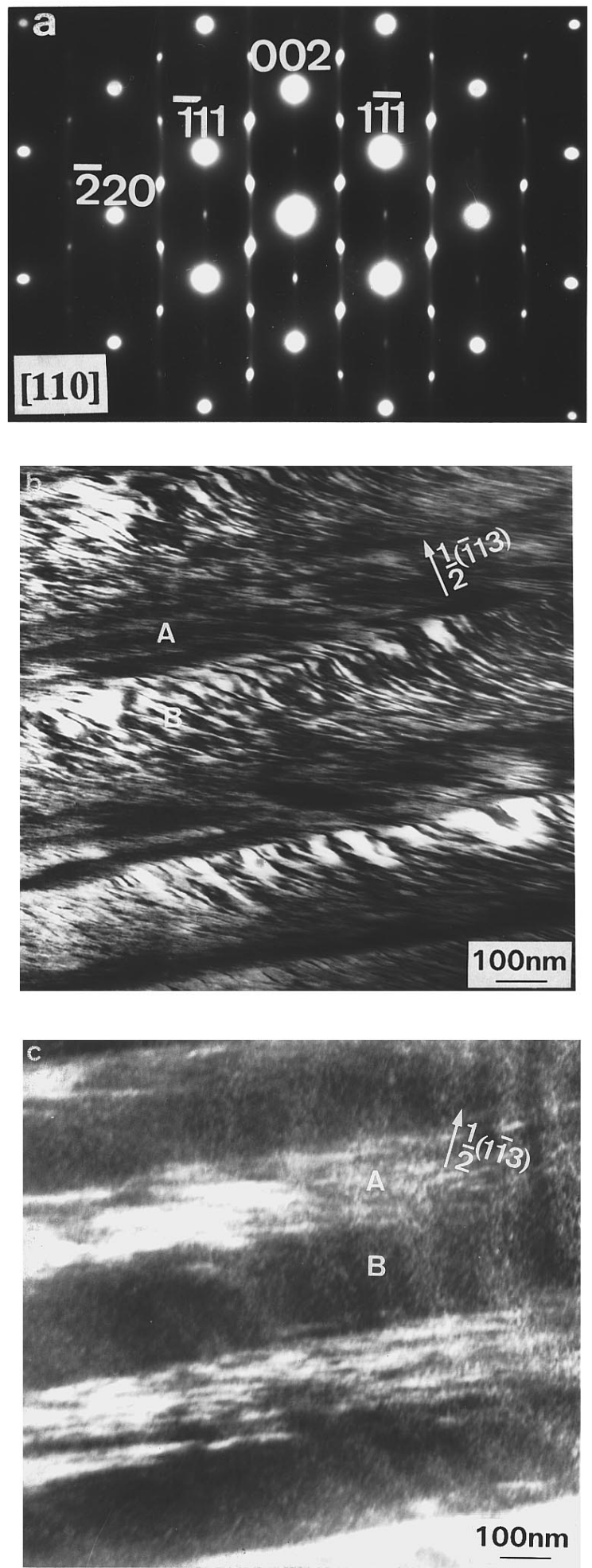

FIG. 1. [110] XTEM view of the sample growth with $\alpha=2^{\circ}$ : (a) SADP exhibiting satellite spots located at $1 / 2(\overline{1} 11)$ and $1 / 2(1 \overline{1} 1)$. Dark-field images taken from (b) the $1 / 2(\overline{1} 13)$ and (c) $1 / 2(1 \overline{1} 3)$.

\section{EXPERIMENTAL DETAILS}

We have analyzed a series of InGaP epilayers $\simeq 4 \mu \mathrm{m}$ thick grown over GaAs substrates cut at tilt angles $\alpha$ of $0^{\circ}$, $2^{\circ}, 5^{\circ}, 10^{\circ}$, and $25^{\circ}$ from the [001] directions towards the [110] direction [the tilt angle of $25^{\circ}$ corresponding then to a
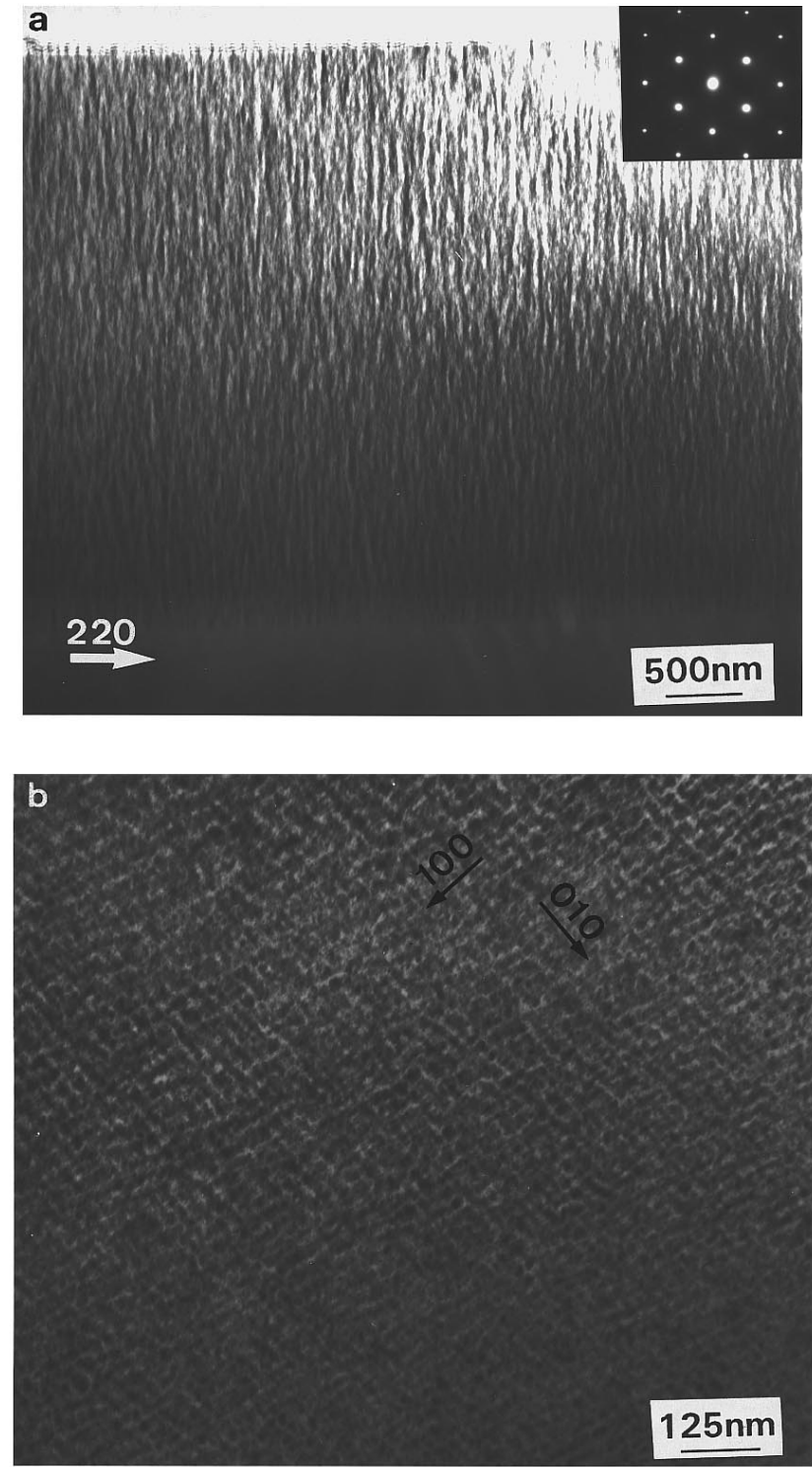

FIG. 2. (a) [110] XTEM of the sample grown with $\alpha=25^{\circ}$ exhibiting a columnar fine contrast modulation close to the [001] direction. The inset shows the SADP without any evidence of ordering. (b) (001) view of the fine contrast modulation oriented along the [100] and [010] directions.

nearby (311) growth surface]. The layers were grown by conventional metal-organic vapor phase epitaxy (MOVPE) in an horizontal geometry reactor with no rotation, at low pressure (150 Torr), at a temperature of $650{ }^{\circ} \mathrm{C}$ and a growth rate of $\simeq 500 \AA / \mathrm{min}$. The sources were the conventional TMG, TMI, and $\mathrm{PH}_{3}$. There were no dopants and the V/III flux ratio was 350 .

The samples were examined by TEM in (001) plan-view and cross-section orientations along both the [110] and [1 $\overline{10}$ ] directions, using a Hitachi H800-NA and a Phillips CM30 SuperTWIN operated at $200 \mathrm{kV}$.

\section{RESULTS}

A first step in studying the evolution of phase separation and ordering is to know the main features of each one of them; so, for the description of each structure we have se- 

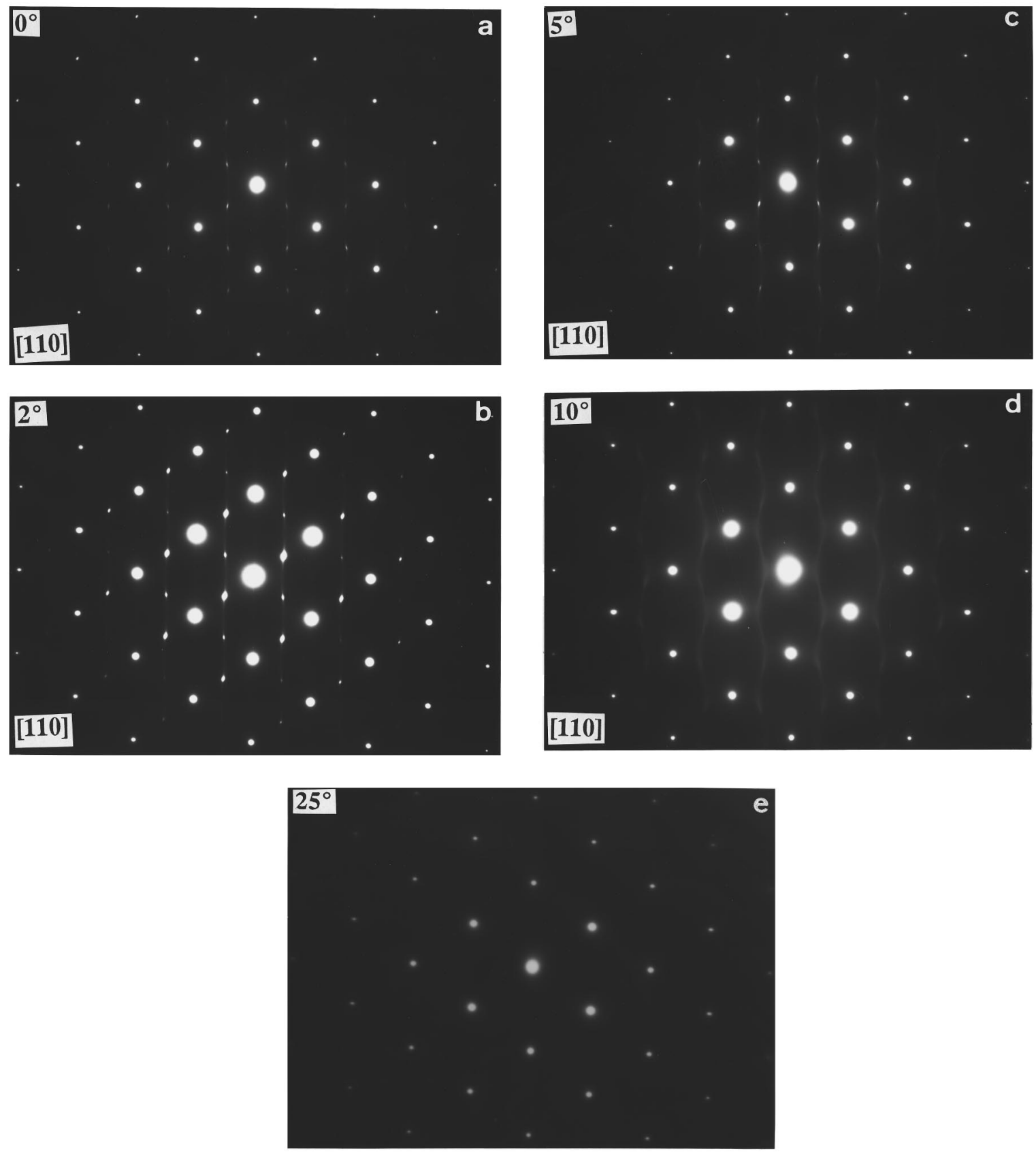

FIG. 3. Evolution of the degree of ordering depending on the misorientation angle: (a) $\alpha=0^{\circ}$; (b) $\alpha=2^{\circ}$; (c) $\alpha=5^{\circ}$; (d) $\alpha=10^{\circ}$; and (e) $\alpha=25^{\circ}$.

lected the most representative sample. Concerning the CuPttype ordering, it is more evident in the sample grown with a misorientation angle of $2^{\circ}$. The selected area diffraction pattern along the [110] zone axis of this sample exhibits two sets of extra diffraction spots, located at $1 / 2(\overline{1} 11)$ and $1 / 2(1 \overline{1} 1)$ [Fig. 1(a)]. Conversely, the observation along the [110] zone axis, only shows the zinc-blende reflections. As reported commonly, the existence of only two of the four possible $\mathrm{CuPt}$ variants is produced by the reduced symmetry of the zinc-blende (001) surface. ${ }^{18,19}$ The presence of these half-order diffraction spots in the [110] TED patterns implies that neighboring (111) planes are not equivalent, with In and Ga having segregated such that $(\overline{1} 11)$ planes of the group-III sublattice are alternately rich in In and $\mathrm{Ga}$, i.e., ordering of the (111) planes has occurred. Figures 1(b) and 1(c) are darkfield (DF) images taken from the $1 / 2(\overline{1} 13)$ and $1 / 2(1 \overline{1} 3)$ superspots, respectively. The DF images show that the epilayer is occupied by domains [labeled A and B in Figs. 1(b) and 1(c)] within which the ordered areas are platelets with typical dimensions of $200 \times 20 \mathrm{~nm}^{2}$. In each domain a single-order variant, either $1 / 2(\overline{1} 11)$ or $1 / 2(1 \overline{1} 1)$, is formed.

As far as phase separation is concerned, it is more clearly observed in the most misoriented sample $\left[25^{\circ}\right.$ off $[001] \rightarrow[110]$, i.e., a nearby (311) growth]. The cross-section view of this sample shows a columnar fine contrast modulation close to the [001] direction [Fig. 2(a)]. The selected area diffraction pattern along both the [110] and [110] zone axis (SADP) [inset in Fig. 2(a)] does not present any evidence of 

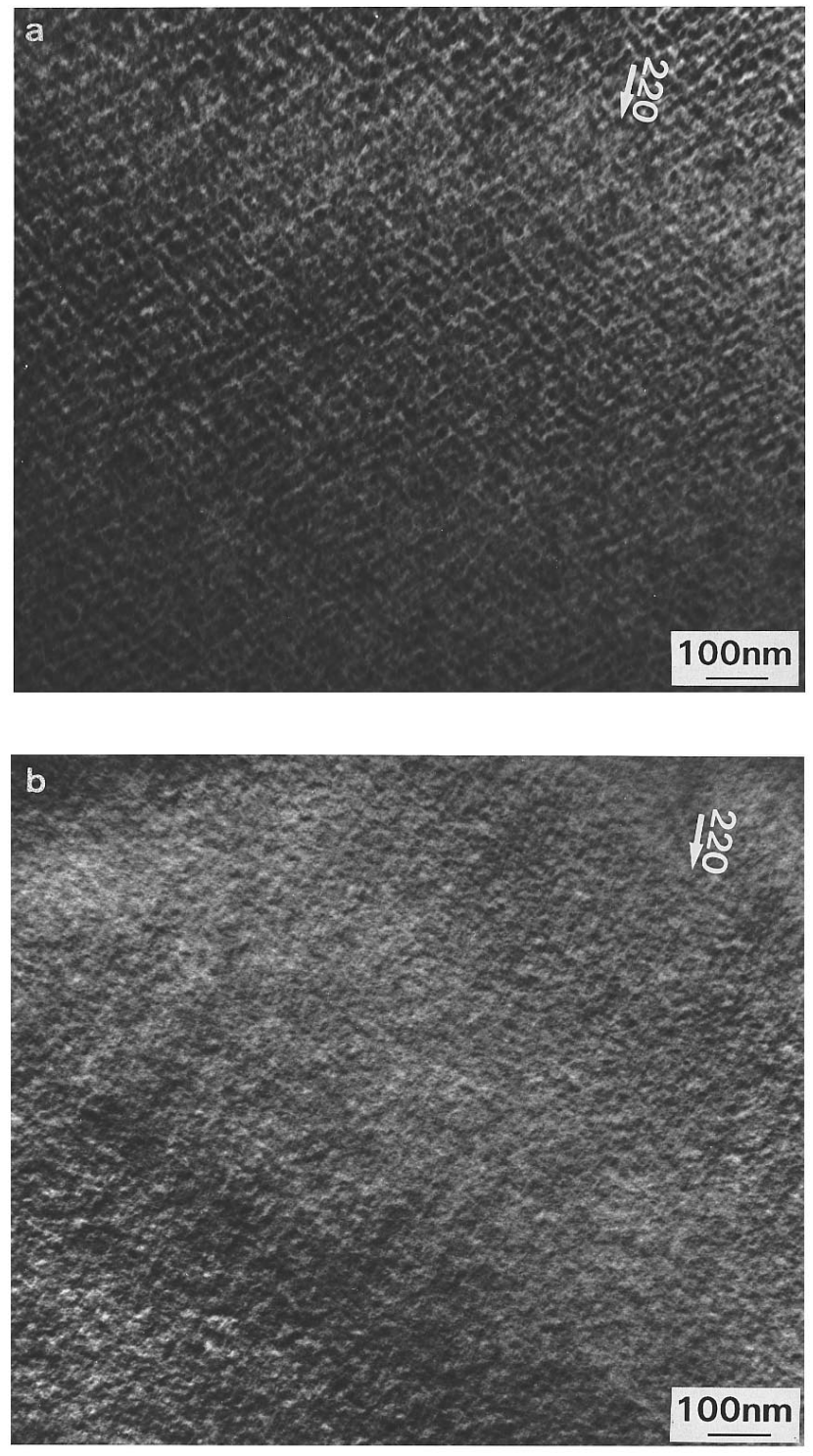

FIG. 4. (001) view of the fine contrast modulation for the samples grown with (a) $\alpha=25^{\circ}$ and (b) $\alpha=2^{\circ}$.

extra spots besides the standard zinc-blende reflections. Planview images present a fine scale quasiperiodic strain contrast oriented along the [100] and [010] directions [Fig. 2(b)]. This contrast modulation along both $\langle 010\rangle$ directions is clearly observable under $g=022$ bright-field two-beam conditions. Conversely, with $g=040$, only dark bands along [001] are visible whereas for $g=004$, lines on [010] remain in strong contrast. The presence of this fine modulation (FM) structure has been observed in many III-V alloys and is attributed to alloy clustering associated with the occurrence of spinodal decomposition. ${ }^{4,5,20,21}$ Although this structure is present in all the samples studied, independently of the substrate misorientation, their contrast varies, being less evident in the sample grown over a $2^{\circ}$ misoriented substrate.

Once the main features have been described, we pass to discuss their evolution with the misorientation angle. Figure 3 illustrates the modification of SADP along the [110] zone axis as the substrate misorientation angle increases. Whereas in the sample grown with $\alpha=0$ the satellite spots are very faint [Fig. 3(a)], their strength is maximum for $\alpha=2^{\circ}$. For increasing $\alpha$ the intensity of the satellite weakens [Fig. 3(c)], and they become just a diffuse intensity along [110] for $\alpha=10^{\circ}$ [Fig. 3(d)] and finally disappear for $\alpha=25^{\circ}$ [Fig. $3(\mathrm{e})]$. The above features point to a very strong sensitivity of the epilayer microstructure to the substrate orientation.

Concomitantly, the fine modulation, present with higher strength in the sample grown with $\alpha=25^{\circ}$ [Fig. 4(a)], also evolves with $\alpha$, in such a way that the more ordered the layer, the less defined the contrast modulation pattern $\left[\alpha=2^{\circ}\right.$, Fig. 4(b)].

In summary, the intensity of the superlattice spots, a measure of the volume fraction and degree of atomic ordering, decreases with increasing the misorientation angle beyond $\alpha=2^{\circ}$. Conversely, the phase separation is more evident when increasing the misorientation angle.

\section{DISCUSSION}

The appearance of phase separation and ordering may be explained from the thermodynamic aspects of the stability of the ternary alloys. The ordering is proposed to arise at the surface of the layer during growth ${ }^{22,23}$ by a process of rapid surface diffusion of the group-III atoms, forming ordered surface monolayers which are then overgrown and frozen into the bulk of the layer. However, since the CuPt-type structure is predicted to be unstable in the bulk, the ordered structure could subsequently disorder in the bulk of the layer during further growth by the slower process of bulk diffusion to lower the free energy state in the bulk. So, two main growth parameters must be taken into account in order to explain the evolution of the structure (and hence, TED patterns): the temperature and the surface misorientation.

For the phase separation, following the valence force field model, ${ }^{24}$ the five tetrahedron units with coordination number between $n=0$ and $n=4$ are not equally probable. Then the adatoms may redistribute when reaching the growing front so as to accommodate the dissimilar bond lengths between In-P and $\mathrm{Ga}-\mathrm{P}$ bonds with the minimum Gibbs free energy. According with the model proposed by Ichimura and $\mathrm{Asaki}^{25}$ in the expression of the Gibbs energy $\Delta F$ $=\Delta H-T \Delta S$, the term $\Delta H$ can be separated in two contributions $\Delta H^{e}+\Delta H_{\alpha \beta}^{t}$, the former corresponding to the energy of bond formation in the binary alloy and the latter related to the additional elastic energy due to the elongation and bending of the bonds in the ternary. Then, if we consider the growth of the ternary as a mixture of the two binary phases $\alpha$ and $\beta$ leading to a compound $\alpha \beta$, the variation of the Gibbs energy of the system should be

$$
\Delta F=\Delta H^{e}+H_{\alpha \beta}-T\left(S_{\alpha \beta}-S_{\alpha+\beta}\right) .
$$

Since $\Delta H$ for pseudobinary formation is high, it dominates over the entropy term at relatively low growth temperatures. Hence, the lower $H_{\alpha \beta}^{t}$ is, the lower $F$ is and therefore the systems tend to reduce the state of bond strain separating the compound toward the respective binaries. This leads to a phase separation by spinodal decomposition at a temperature below the critical value $T_{c}$. In these conditions the system might stabilize with a certain degree of mixing between the 
constituents. As shown by Ferreira, Wei, and Zunger, ${ }^{26}$ the state of minimum energy corresponds to a nonrandom distribution of tetrahedron with different $n$ values, avoiding a complete phase separation.

On the other hand, the evolution of the long-range ordering and phase separation with the misorientation substrate suggests that the presence of the steps at the surface when growing the layers on vicinal surfaces plays an important role on the stability of ordered phases. Consequently, the influence of a surface energy term has to be taken into account when analyzing the stability of the system. So, the effect of the presence and motion of the [110] surface steps has been taken into account in the model proposed by Philips et al. ${ }^{18}$ to explain the preferential formation of the (111) and (111) variants on vicinal (001) surfaces offcut a few degrees toward the [110] direction. In the first part of this model the authors assume that, when growing the layers under group- $\mathrm{V}$ rich surface conditions, the dimerization of the surface atoms induce subsurface strains conditioning the incorporation of the larger group-III atoms (In in our case) in the atomic subsurface sites under tension and the smaller group-III atoms $(\mathrm{Ga})$ in the sites under compression. When introducing these proposals on the valence force field calculations, ${ }^{18}$ the results show a reduction of the strain energy by $100 \mathrm{meV} /$ dimer site over the less favorable arrangements. In the second part of the model they consider that the lowest-energy configuration corresponds to the presence of terraces between steps containing an even number of surface group- $\mathrm{V}$ atoms, because in this case all the group- $\mathrm{V}$ atoms on the terraces between steps can form dimers. This step-terrace reconstruction of the surface favors the development of the ordering only on the $(\overline{1} 11)$ and ( $1 \overline{1} 1)$ variants. If a terrace of width corresponding to an odd number of group- $\mathrm{V}$ atoms was present on the reconstructed surface this could lead to the formation of an antiphase boundary (APB) in the ordered structure. Recently, Su and Stringfellow ${ }^{27}$ have analyzed the effect of the presence of supersteps on the ordering, showing that:

there is near equality between APB and superstep spacing (space that increases when increasing misorientation angle) suggesting that supersteps result in the formation of APB;

(ii) the size of the (001) facets developing on the surface of the largest supersteps during the growth is independent of the misorientation angle suggesting a surface diffusion limited size.

In the range of temperatures where our samples have been grown, the occurrence of phase separation is expected to be the most stable configuration of the system. However, the reduction on strain energy induced by the group- $\mathrm{V}$ surface reconstruction and the presence of steps favor the stability of large ordered structures. These ordered structures are present in two of the four (111) variants possible and they are more evident in the case of the $2^{\circ}$ misoriented substrates, since, in this case, a more uniform distribution of monolayer steps separated by terraces with an even number of atoms in the surface will exist. This distribution could favor the development of extended ordered regions in all the surface. In the case of more misoriented substrates, both the height of the supersteps and the length of terraces between them increase. This gives rise to a loss of the surface reconstruction coherence that would explain why extended ordered domains do not develop. In this situation the phase separation becomes again the most stable configuration for the epitaxial layer.

\section{CONCLUSIONS}

We have described the competitive evolution of the phase separation and ordering developed in InGaP layers grown on misoriented GaAs substrates depending on the tilt angle. The most ordered configuration has been found to appear in layers grown on $2^{\circ}$ off substrates, and the strength of order decreases with increasing the misorientation angle beyond $\alpha=2^{\circ}$. Conversely, whereas the phase separation is less evident in the layer grown at $2^{\circ}$, the sample grown with a misorientation of $25^{\circ}$ exhibits the most phase separated configuration. Although in the range of temperature where our samples have been grown the occurrence of phase separation is expected to be the most stable configuration of the system, the reduction on strain energy induced by the group- $\mathrm{V}$ surface reconstruction and the presence of steps favor the stability of large ordered structures. For high misorientation angles, the loss of coherence of surface reconstruction at steps explains why the development of extended domains is less probable and, therefore, why the phase separated structure becomes again the most stable configuration.

\section{ACKNOWLEDGMENTS}

The authors would like to acknowledge R. W. Glew for the growth of the samples. This work has been partially supported by the Spanish programs MAT93-0564 and the DGICYT Project No. PB94-0719.

${ }^{1}$ B. de Cremoux, J. Phys. (Paris) Colloq. 43, C5 (1982).

${ }^{2}$ S. H. Wei, L. G. Ferreira, and A. Zunger, Phys. Rev. B 41, 8240 (1990).

${ }^{3}$ S. Mahajan, M. A. Shahid, and D. E. Laughlin, Institute of Physics Conference Series, Vol. 100, edited A. G. Cullis and J. L. Hutchinson (IOP, 1989), p. 143.

${ }^{4}$ F. Glas, Inst. Institute of Physics Conference Series, edited by A. G. Cullis, A. E. Staton-Bevan, and J. L. Hutchison, Vol. 134 (IOP, 1993), p. 269.

${ }^{5}$ F. Peiró, A. Cornet, A. Herms, J. R. Morante, S. A. Clark, and R. H. Williams, J. Appl. Phys. 73, 4319 (1993).

${ }^{6}$ G. P. Srivastava, J. L. Martins, and A. Zunger, Phys. Rev. B 31, 2561 (1985).

${ }^{7}$ I. J. Murgatroyd, A. G. Norman, G. R. Booker, and T. M. Kerr, J. Electron. Microsc. 35, 1497 (1986).

${ }^{8}$ A. G. Norman, T.-Y. Seong, B. A. Philips, G. R. Booker, and S. Mahajan, Inst. Phys. Conf. Ser. 134, 279 (1993).

${ }^{9} \mathrm{~J}$. W. Christian, The Theory of Transformations in Metals and Alloys (Pergamon, New York, 1975), Part I.

${ }^{10}$ H. Nakayama and H. Fujita, GaAs and Related Compounds 1985, Inst. Phys. Conf. Ser., Vol. 79, edited by M. Fujimoto (IOP, 1986), p. 289.

${ }^{11}$ T. S. Kuan, W. I. Wang, and E. L. Wilkie, Appl. Phys. Lett. 51, 51 (1987).

${ }^{12}$ M. A. Shahid, S. Mahajan, D. E. Laughlin, and H. M. Cox, Phys. Rev. Lett. 58, 2567 (1987).

${ }^{13}$ I. J. Murgatroyd, A. G. Norman, and G. R. Booker, J. Appl. Phys. 67, 2310 (1990).

${ }^{14}$ P. Bogulawski, Phys. Rev. B 42, 3737 (1990).

${ }^{15}$ S. Froyen and A. Zunger, Phys. Rev. Lett. 66, 2132 (1991). 
${ }^{16}$ S. Froyen, J. E. Bernard, R. Osorio, and A. Zunger, Phys. Scr. T45, 272 (1992).

${ }^{17}$ J. E. Bernard, S. Froyen, and A. Zunger, Phys. Rev. B 44, 111178 (1991).

${ }^{18}$ B. A. Philips, A. G. Norman, T. Y. Seong, S. Mahajan, G. R. Booker, M. Skowronski, J. P. Harbison, and V. G. Keramidas, J. Cryst. Growth 140, 249 (1994).

${ }^{19}$ L. C. Su, S. T. Pu, G. B. Stringfellow, J. Christen, H. Selber, and D. Bimberg, J. Electron. Mater. 23, 125 (1994).

${ }^{20}$ T. Y. Seong, G. R. Booker, and A. G. Norman, Microscopy of Semiconducting Materials 1993, Inst. Phys. Conf. Ser., Vol. 134, edited by A. G. Cullis, A. E. Staton-Beran, and J. L. Hutchison (IOP, 1993), p. 301.
${ }^{21}$ F. Peiró, A. Cornet, P. Roura, and J. R. Morante, Mater. Res. Soc. Symp. Proc. 379, 159 (1995).

${ }^{22}$ P. Bellon, J. P. Chevalier, E. Augarde, J. P. André, and G. P. Martin, J. Appl. Phys. 66, 2388 (1989).

${ }^{23}$ S. R. Kurtz, J. M. Olson, and A. Kibbler, Appl. Phys. Lett. 57, 1922 (1990).

${ }^{24}$ J. B. Joyce and J. C. Mikkelsen, Jr., J. Cryst. Growth 98, 18 (1989).

${ }^{25}$ M. Ichimura and A. Asaki, J. Cryst. Growth 98, 18 (1989).

${ }^{26}$ L. G. Ferreira, S. H. Wei, and A. Zunger, Phys. Rev. B 40, 3197 (1989).

${ }^{27}$ L. C. Su and G. B. Stringfellow, J. Appl. Phys. 78, 6775 (1995). 